\title{
Antibiotic susceptibility of coagulase-negative staphylococci (CoNS): emergence of teicoplanin- non-susceptible CoNS strains with inducible resistance to vancomycin
}

\author{
Correspondence \\ Xiao Xue Ma \\ xxma@mail.cmu.edu.cn
}

Received 6 May 2011

Accepted 21 July 2011

\author{
Xiao Xue Ma, ${ }^{1}$ En Hua Wang, ${ }^{2}$ Yong $\mathrm{Liu}^{3}$ and En Jie Luo ${ }^{1}$ \\ 1Department of Medical Microbiology and Parasitology, College of Basic Medical Sciences, \\ China Medical University, Shenyang, PR China \\ ${ }^{2}$ Institute of Pathology and Pathophysiology, China Medical University, Shenyang, PR China \\ ${ }^{3}$ Department of Clinical Microbiology, Second Affiliated Hospital of China Medical University, \\ Shenyang, PR China
}

\begin{abstract}
Coagulase-negative staphylococci (CoNS) have become increasingly recognized as important agents of nosocomial infection. One of the characteristics of CoNS is their resistance to multiple antimicrobial agents commonly used for the treatment of staphylococcal infections. CoNS strains $(n=745)$ isolated from a university teaching hospital in China between 2004 and 2009 were tested for antibiotic resistance. The antibiotics were placed into three categories based on resistance levels of the CoNS strains to these antibiotics: high resistance (resistance rate $>70 \%$ ), including penicillin $\mathrm{G}$, oxacillin and erythromycin; medium resistance (resistance rate between 30 and $70 \%$ ), including tetracycline, clindamycin, ciprofloxacin, trimethoprim/ sulfamethoxazole and chloramphenicol; and low resistance (resistance rate $<30 \%$ ), including rifampicin, ceftizoxime and gentamicin. We also found that the prevalence of strains nonsusceptible to teicoplanin increased from 4.5 to $6.7 \%$ between 2008 and 2009. A one-step vancomycin agar selection experiment and subsequent population analysis revealed potentially vancomycin-resistant subpopulations that have been selected from the teicoplanin-nonsusceptible strains. Vigilant surveillance of nosocomial isolates of CoNS is needed to determine their resistance to glycopeptides.
\end{abstract}

\section{INTRODUCTION}

Coagulase-negative staphylococci (CoNS) are among the most frequently isolated micro-organisms in clinical microbiology laboratories (Cervera et al., 2009; Hung et al., 2011). Since the 1970s, it has been suggested that CoNS are of great importance as true pathogens, and they have become a common cause of a wide variety of infections (Dubois et al., 2010; Otto, 2009). Infections with CoNS occur mainly in specific groups of patients, including patients with neutropenia, neonates and patients with indwelling foreign devices (Huebner \& Goldmann, 1999; Zong et al., 2011). Infections at metastatic sites, such as the central nervous system, heart, bones and joints, are especially common, and such infections in these vulnerable populations are difficult to treat (Casey et al., 2007).

The frequency of oxacillin resistance in CoNS strains has increased substantially over recent decades (Stefani \&

Abbreviations: CoNS, coagulase-negative staphylococci; TMP/SMX, trimethoprim/sulfamethoxazole; VRSA, vancomycin-resistant Staphylococcus aureus.
Varaldo, 2003). This has led to the frequent use of glycopeptide antibiotics for treatment of CoNS infections (Ferreira et al., 2003; Srinivasan et al., 2002; Tacconelli et al., 2001). As a result, the emergence of strains with decreased levels of susceptibility to vancomycin and teicoplanin has been reported (Cremniter et al., 2010; Trueba et al., 2006). Since the 1990s, a worldwide increase in the number of observations of glycopeptide-resistant CoNS has been described (Bourgeois et al., 2007). Some recent reports have pointed out reduced susceptibility of glycopeptides within Staphylococcus epidermidis in various European countries, suggesting that such strains might be highly disseminated in the community and in hospitals (Cremniter et al., 2010; Gazzola \& Cocconcelli, 2008; Maniati et al., 2005; Natoli et al., 2009; Trueba et al., 2006).

In this report, we systematically studied the antibiotic resistance of all CoNS strains collected between 2004 and 2009 at the Department of Clinical Microbiology at the Second Affiliated Hospital of China Medical University, which is a 4300-bed university teaching hospital. We also performed an epidemiological study of the teicoplanin-resistant CoNS 
strains. The purpose of the study is to provide insight toward our understanding of glycopeptide resistance of CoNS and to evaluate its clinical significance.

\section{METHODS}

CoNS strains. In total, 745 CoNS strains were used in this study. The strains were collected between 2004 and 2009 from different clinical specimens, including blood $(47.3 \%)$, nostrils (15.8\%), wound $(10.5 \%)$, urine $(7.9 \%)$, catheter tips $(2.6 \%)$, drainage fluids $(5.4 \%)$, sputum $(4.7 \%)$, and secretions in general (5.8\%). Only one strain from each patient was analysed. The CoNS strains were collected from the following departments of the Second Affiliated Hospital of China Medical University: internal medicine (including cardiology, $n=49$; neurology, $n=61$; nephrology, $n=39$; haematology, $n=54$; gastroenterology, $n=66$; endocrinology, $n=36$; rheumatology, $n=23$; respiratory medicine, $n=37)$, the surgical department $(n=114)$, surgery intensive care $(n=78)$, neurosurgery $(n=53)$, thoracic surgery $(n=61)$ and paediatrics $(n=74)$. The CoNS strains used in this study were either isolated from clinically significant materials (e.g. blood or normally sterile fluids) in the presence of clinical manifestations not attributable to other causes, or isolated from samples in the presence of clinical evidence of infection not attributable to other micro-organisms. Strains were named in Arabic numerals in accordance with the time collected. The staphylococcal strains were first identified by using Pos Combo type 4I MicroScan panels (Baxter Diagnostics). They were further identified and confirmed by using the API 20 Staph system (bioMérieux). The strains were stored at $-20{ }^{\circ} \mathrm{C}$ in tryptic soy broth containing $20 \%$ (v/v) glycerol.

Susceptibility tests. The broth microdilution technique was used for initial antimicrobial susceptibility testing, for which customized broth microdilution panels manufactured by Microscan combo ID/ AST (Baxter Diagnostics) were used. A suspension with a density equivalent to that of a $0.5 \mathrm{McF}$ arland standard inoculum was prepared in $0.9 \%$ saline to achieve a final density of approximately $5 \times 10^{5}$ c.f.u. $\mathrm{ml}^{-1}$. MICs were read as the lowest concentration at which growth was inhibited. The antimicrobial agents tested included penicillin G, oxacillin, rifampicin, tetracycline, chloramphenicol, ceftizoxime, ciprofloxacin, clindamycin, erythromycin, gentamicin, trimethoprim/sulfamethoxazole (TMP/SMX), teicoplanin and vancomycin. We used the agar dilution method to further confirm the teicoplanin and vancomycin MIC values of the teicoplanin-nonsusceptible CoNS isolates. The procedure was performed in duplicate on separate occasions, and the means of the duplicates were used. Staphylococcus aureus ATCC 29213 was used as the quality control in each set of tests.

Population analysis of CoNS strains. Analysis of the strain subpopulations resistant to vancomycin and teicoplanin was performed. In brief, strains were first cultured overnight in brain-heart infusion (BHI) broth. Then, $50 \mu \mathrm{l}$ bacterial suspension was transferred to $4 \mathrm{ml}$ pre-warmed BHI broth and incubated at $37{ }^{\circ} \mathrm{C}$ until the $\mathrm{OD}_{578}$ was approximately 0.7 . After the $\mathrm{OD}_{578}$ was adjusted to 0.3 (approx. $10^{8}$ c.f.u. $\mathrm{ml}^{-1}$ ), 10 -fold dilutions of cell suspensions were used to inoculate BHI agar plates containing $0,1,2,3,4,5,6,7$, $8,9,10,11$ or $12 \mathrm{mg}$ vancomycin $1^{-1}$, or $0,0.5,1,2,4,8,16,32,64$, 128 or $256 \mathrm{mg}$ teicoplanin $1^{-1}$. The plates were incubated at $37^{\circ} \mathrm{C}$ for $48 \mathrm{~h}$, and then the colonies were counted. Heterogeneous vancomycin-resistant $S$. aureus (hetero-VRSA) Mu3 and intermediate VRSA Mu50 were used as quality controls.

One-step selection of vancomycin-resistant CoNS strains. To screen for potentially vancomycin-resistant strains among teicopla- nin-non-susceptible CoNS strains, we carried out a one-step resistance selection experiment by using vancomycin agar. Vancomycin agar was prepared by incorporating 4 or $6 \mu \mathrm{g}$ vancomycin $\mathrm{ml}^{-1}$ into BHI agar. Agar was poured into $85 \mathrm{~mm}$ Petri dishes, with a $4 \mathrm{~mm}$ depth of agar. Overnight cultures in BHI broth were adjusted to an $\mathrm{OD}_{578}$ of $0.3\left(10^{8}\right.$ c.f.u. $\left.\mathrm{ml}^{-1}\right)$. Aliquots $(10 \mu \mathrm{l})$ of the cell suspensions were placed evenly onto the vancomycin agar. The plates were then incubated at $37{ }^{\circ} \mathrm{C}$ for $48 \mathrm{~h}$, after which time cell growth was inspected.

PCR amplification of van and mecA genes. Chromosomal DNA was prepared by the small-scale phenol extraction method and used as PCR template (Matsuhashi et al., 1986). PCRs were performed using a Gene Amp 9600 thermal cycler (Perkin-Elmer Cetus Instruments). All strains were subjected to $m e c A, \operatorname{van} A, \operatorname{van} B$ and $\operatorname{van} C$ gene $\mathrm{PCR}$ with primers described previously (Kristóf et al., 2011; Ma et al., 2011). DNA amplification was performed using the following cycling parameters: denaturation at $94{ }^{\circ} \mathrm{C}$ for $2 \mathrm{~min}$, followed by 30 amplification cycles of $1 \mathrm{~min}$ at $94{ }^{\circ} \mathrm{C}, 1 \mathrm{~min}$ at $52{ }^{\circ} \mathrm{C}$ and $1 \mathrm{~min}$ at $72{ }^{\circ} \mathrm{C}$, with a final extension step at $72{ }^{\circ} \mathrm{C}$ for $10 \mathrm{~min}$. The resulting fragments were electrophoresed in $1.5 \%$ agarose gel, stained with ethidium bromide and visualized under UV light. Quality-control strains included S. aureus N315 (mecA), Enterococcus faecalis NCTC 12201 (vanA), Enterococcus faecalis ATCC 51299 (vanB), Enterococcus gallinarum NCTC $12359^{\mathrm{T}}$ (vanC1) and Enterococcus casseliflavus NCTC $12361^{\mathrm{T}}$ (vanC2).

Statistical analysis. SPSS for Windows (version 12.0; SPSS) software was used for the analysis. Categorical variables were compared by using $\chi^{2}$ or Fisher's exact probability tests. $P$-values were calculated and $P \leqslant 0.05$ was considered statistically significant.

\section{RESULTS AND DISCUSSION}

\section{Antibiotic-resistance profiles of CoNS strains}

Resistance profiles of the CoNS strains against the 13 antibiotics tested are presented in Table 1. In total, 745 CoNS strains were isolated during the study period. Eightynine isolates were collected in 2004, 108 in 2005, 129 in 2006, 121 in 2007, 133 in 2008 and 165 in 2009. The mean resistance rates to penicillin $\mathrm{G}$, oxacillin and erythromycin among all tested CoNS strains were high, at 94.2, 79.1 and $89.5 \%$, respectively. In contrast, the mean resistance rates to rifampicin, ceftizoxime and gentamicin were low, at 18.4, 26.7 and $29.5 \%$, respectively. The resistance rates to tetracycline, clindamycin, TMP/SMX, ciprofloxacin and chloramphenicol were medium, at 59.5, 53.7, 58.5, 52.8 and $39.1 \%$, respectively. It was noteworthy that, from 2004 to 2009, all CoNS strains were susceptible to vancomycin. No strains were detected to be non-susceptible to teicoplanin from 2004 to 2007. Six of the 133 isolates $(4.5 \%)$ collected in 2008 were non-susceptible to teicoplanin (MICs between 16 and $128 \mathrm{mg} \mathrm{l}^{-1}$ ), whereas in 2009 the number of non-susceptible CoNS strains was 11 out of $165(6.7 \%)$. These results show that resistance to teicoplanin in CoNS appeared in 2008 and increased from 2008 to 2009. It has been reported that the meticillin resistance of Staphylococcus haemolyticus is due to the presence of the mecA gene (Zong et al., 2011). The mecA gene may also spread to other meticillin-susceptible $S$. 
Table 1. Resistance rates of CoNS strains to various antibiotics

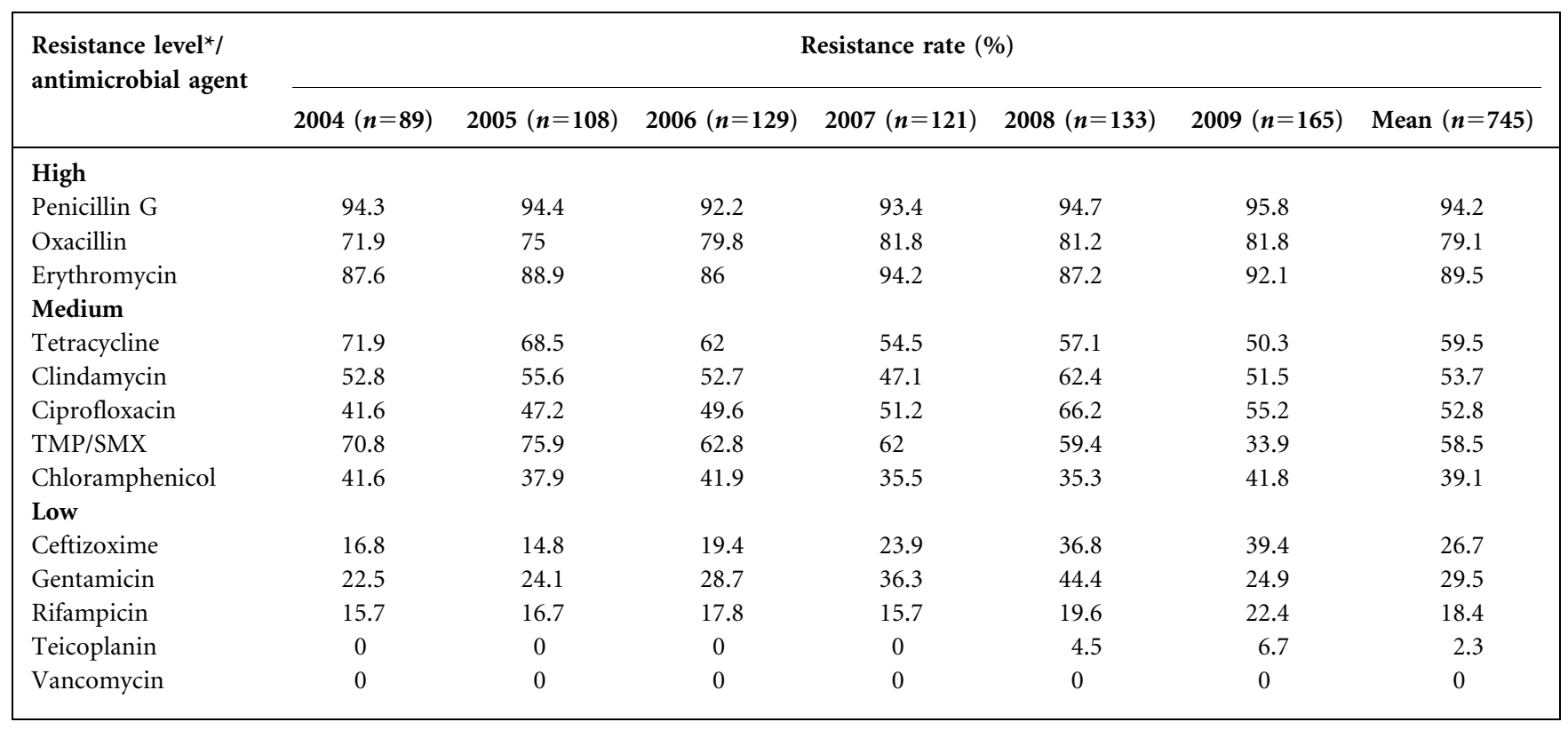

${ }^{\star}$ Resistance level was defined as high if $>70 \%$, medium if $30-70 \%$ and low if $<30 \%$.

haemolyticus strains via transposition (Boyce, 2004). mecA PCR was performed on all strains to confirm resistance of strains phenotypically resistant to methyl penicillins. The results showed that the mecA gene was present in all CoNS strains with oxacillin resistance observed by using phenotypic techniques.

In addition, we analysed the annual change in resistance rate for the CoNS strains to the examined antimicrobial agents over 6 years. As shown in Table 1, we observed that, for penicillin $\mathrm{G}$, oxacillin, erythromycin, clindamycin and chloramphenicol, the annual proportion of resistant CoNS strains did not change significantly from 2004 to 2009. On the other hand, there was a gradual increase in the prevalence of CoNS strains resistant to rifampicin and ciprofloxacin. In contrast, the initial resistance rates of CoNS strains to tetracycline and TMP/SMX in 2004 were high, at 71.9 and $70.8 \%$, respectively, but decreased to 50.3 and $33.9 \%$, respectively, in 2009. Although there were no isolates resistant to vancomycin, six isolates in 2008 and 11 isolates in 2009 were resistant to teicoplanin according to the criteria of the Clinical and Laboratory Standards Institute (CLSI, 2010).

\section{Identification of CoNS species and their multiple-resistance profiles}

Using several different techniques, we identified and confirmed that the 745 CoNS strains belong to 11 species (Table 2). To test whether the antibiotic resistance was correlated with the CoNS species, we compared the resistance profile to multiple antimicrobial agents among the CoNS species between 2004 and 2009. From all assays carried out, both S. haemolyticus and S. epidermidis had high-resistance profiles, as shown in Fig. 1. The percentage of multipleresistant strains, however, did not change significantly in $S$. epidermidis between 2004 and 2009; it did change significantly in S. haemolyticus ( $P=0.04$ ), and Staphylococcus capitis $(P=0.047)$. Furthermore, we found no multiple-resistance profiles in strains of Staphylococcus auricularis, Staphylococcus sciuri, Staphylococcus simulans or Staphylococcus xylosus in 2004. However, multiple-resistance profiles were identified in 2009 with rates of 4.7, 2.8, 2.3 and $1.3 \%$, respectively, among the four species. It was noted that the multipleresistance profile of Staphylococcus saprophyticus decreased significantly during the study period $(P=0.034)$. Resistance

Table 2. CoNS species identified

We identified and confirmed that the 745 CoNS strains belonged to 11 species, using several different techniques as described in Methods.

\begin{tabular}{|lc|}
\hline Species & No. of strains (\%) \\
\hline S. haemolyticus & $254(34.1)$ \\
S. epidermidis & $204(27.4)$ \\
S. simulans & $66(8.9)$ \\
S. hominis subsp. hominis & $53(7.1)$ \\
S. warneri & $43(5.7)$ \\
S. capitis & $31(4.1)$ \\
S. sciuri & $28(3.8)$ \\
S. auricularis & $24(3.2)$ \\
S. saprophyticus & $16(2.1)$ \\
S. caprae & $15(2.0)$ \\
S. xylosus & $11(1.5)$ \\
\hline
\end{tabular}




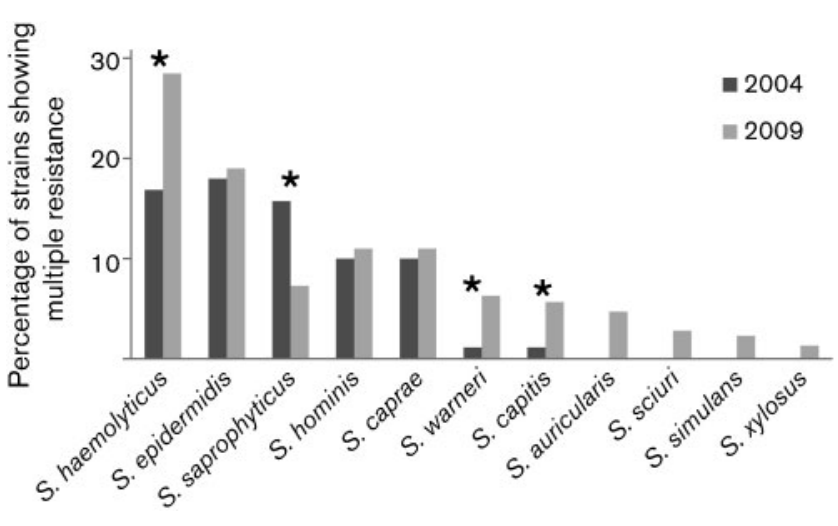

Fig. 1. Comparison of multiple-resistance profiles among different CoNS species between 2004 and 2009. ${ }^{*} P \leqslant 0.05$.

profiles per major species of the CoNS strains against 13 antimicrobial agents were assayed. Resistance to erythromycin was found in

$79 \%$ of S. simulans, $88 \%$ of S. epidermidis and $89 \%$ of S. haemolyticus isolates. Resistance to tetracycline was observed in $64 \%$ of S. epidermidis and $57 \%$ of S. haemolyticus isolates; $44 \%$ of the latter were resistant to ciprofloxacin, whereas S. simulans showed low resistance i.e. $<14 \%$ of strains were resistant. S. hominis showed high susceptibility to tetracycline, clindamycin, ciprofloxacin, TMP/SMX, chloramphenicol and rifampicin.

\section{Screening and characterization of teicoplanin- non-susceptible CoNS strains}

As shown in Table 3, among the six CoNS strains isolated in 2008, the teicoplanin MICs were $64 \mu \mathrm{g} \mathrm{ml}^{-1}$ (S. epidermidis, $n=1$; S. haemolyticus, $n=1$; S. hominis, $n=1$ ) and $16 \mu \mathrm{g} \mathrm{ml}^{-1}$ (S. epidermidis, $n=2 ; S$. haemolyticus, $n=1)$. Among the 11 CoNS strains isolated in 2009, the teicoplanin MICs were $128 \mu \mathrm{g} \mathrm{ml}^{-1}$ (S. epidermidis, $n=2$; S. haemolyticus, $n=2$; S. caprae, $n=1 ; S$. warneri, $n=1 ; S$. hominis, $n=1$ ), $64 \mu_{\mathrm{g} \mathrm{ml}}^{-1}$ (S. epidermidis, $n=1$; S. capitis, $n=1$; S. hominis, $n=1$ ) and $32 \mu \mathrm{g} \mathrm{ml}^{-1}$ (S. haemolyticus, $n=1)$. The result indicated that seven of the 11 CoNS strains $(63.6 \%)$ isolated in 2009 showed a higher level of teicoplanin resistance $\left(\mathrm{MIC}=128 \mu \mathrm{g} \mathrm{ml}^{-1}\right)$. MICs for vancomycin of all the isolates were $\leqslant 2 \mu \mathrm{g} \mathrm{ml}^{-1}\left(2 \mu \mathrm{g} \mathrm{ml}^{-1}\right.$ for nine strains, $1 \mu \mathrm{g} \mathrm{ml}^{-1}$ for five strains and $0.5 \mu \mathrm{g} \mathrm{ml}^{-1}$ for three strains).

To screen for potentially vancomycin-resistant strains among the 17 teicoplanin-non-susceptible CoNS strains, we carried out a one-step resistance selection experiment using vancomycin-containing agar. We found that all CoNS strains could grow on selection agar with $4 \mu \mathrm{g}$ vancomycin $\mathrm{ml}^{-1}$. However, only strains CoNS25 (S. haemolyticus), CoNS06 (S. epidermidis), CoNS29 (S. epidermidis), CoNS74 (S. haemolyticus), CoNS102 (S. warneri), CoNS104 (S. hominis) and CoNS144 (S. hominis) could grow on selection agar containing $6 \mu \mathrm{g}$ vancomycin $\mathrm{ml}^{-1}$. The MIC values of the subpopulation of CoNS strains that could grow on the $6 \mu \mathrm{g}$ vancomycin $\mathrm{ml}^{-1}$ selection agar were determined by the agar dilution method. The results indicated that the MIC values for teicoplanin in these strains did not change significantly. However, the MIC values for vancomycin in six of the seven $(85.7 \%)$ strains growing on the $6 \mu \mathrm{g}$ vancomycin $\mathrm{ml}^{-1}$ agar were $\geqslant 4 \mu \mathrm{g} \mathrm{ml}^{-1}$.

It has been reported that the $v a n A, v a n B$ and $v a n C$ genes are related to acquired glycopeptide resistance in enterococci. vanA can induce resistance to high levels of both vancomycin and teicoplanin (Lorenzo-Díaz et al., 2004; Périchon \& Courvalin, 2009), while vanB induces resistance to various levels of vancomycin only (Lorenzo-Díaz et al., 2004). vanC was characterized by its low-level resistance to vancomycin (Tschudin Sutter et al., 2010). Our PCR results showed that none of the 17 teicoplanin-non-susceptible strains carried the vanA, vanB or vanC genes.

The CLSI (2010) recommended that all staphylococcal isolates should have their MICs tested, and CoNS strains should be evaluated further by a reference centre if the vancomycin MIC is $\geqslant 32 \mu \mathrm{g} \mathrm{ml}^{-1}$. The term 'heteroresistance' to vancomycin has been used for staphylococcal isolates that contain a cell population with different levels of vancomycin susceptibility, including vancomycin-resistant cells (Palazzo et al., 2005). Population analysis of the representative strains CoNS25 (S. haemolyticus), CoNS06 ( $S$. epidermidis), CoNS29 (S. epidermidis), CoNS102 (S. warneri), CoNS104 (S. hominis) and CoNS144 (S. hominis) in the presence of vancomycin or teicoplanin was performed.

Fig. 2 illustrates the population curves of the six CoNS strains that could grow on agar containing $6 \mu \mathrm{g}$ vancomycin $\mathrm{ml}^{-1}$. Their vancomycin MICs were $\geqslant 4 \mu \mathrm{g} \mathrm{ml}^{-1}$, similar to the control strains VRSA Mu50 and heteroVRSA Mu3. CoNS25, CoNS06, CoNS29 and CoNS144 showed a similar type of heteroresistance to vancomycin as $\mathrm{Mu} 3$. CoNS102, with a vancomycin MIC of $6 \mu \mathrm{g} \mathrm{ml}^{-1}$, and CoNS104, with a vancomycin MIC of $8 \mu \mathrm{g} \mathrm{ml}^{-1}$, harboured a subpopulation of cells that could grow on agar plates containing $8 \mu \mathrm{g}$ vancomycin $\mathrm{ml}^{-1}$ at a frequency of $>0.1$ and $0.01 \%$, respectively, suggesting a similar type of intermediate vancomycin resistance to Mu50.

Demographic and clinical characteristics of the patients in which the CoNS strains were non-susceptible to teicoplanin are summarized in Table 3. Among the 17 patients, ten were male and seven were female. Their ages ranged from 12 days to 82 years, and $12(70.6 \%)$ of the infected patients had been treated previously with vancomycin. After adequate treatment, five patients recovered from the infections, seven became afebrile and five died. None of the patients had been treated previously with teicoplanin. It is notable that eight of the 12 patients received vancomycin and $\beta$-lactam antibiotics concomitantly at the time of the underlying disease. It has been speculated that the emergence of staphylococci with heteroresistance to vancomycin could be related to the common practice of 
Table 3. Characteristics of 17 teicoplanin-non-susceptible CoNS strains and clinical characteristics of the patients from whom CoNS strains were isolated

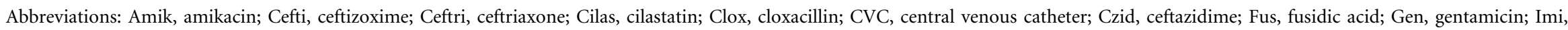
imipenem; Mero, meropenem; Mtz, metronidazole; Pip, piperacillin; Pipe, piperacillin; Rif, rifampicin; SSI, surgical-site infection; Taz, tazobactam; Tei, teicomycin; Van, vancomycin.

\begin{tabular}{|c|c|c|c|c|c|c|c|c|c|c|c|c|c|}
\hline \multicolumn{9}{|c|}{ Strain information } & \multicolumn{5}{|c|}{ Patient information } \\
\hline \multirow[t]{2}{*}{ Strain no. } & \multirow[t]{2}{*}{$\begin{array}{l}\text { Year of } \\
\text { isolation }\end{array}$} & \multirow[t]{2}{*}{ Species } & \multicolumn{2}{|c|}{ MIC } & \multicolumn{2}{|c|}{$\begin{array}{l}\text { Growth on agar } \\
\text { containing } \\
\text { vancomycin at: }\end{array}$} & \multicolumn{2}{|c|}{$\begin{array}{c}\text { MIC from } \\
\text { subpopulation } \\
\text { on vancomycin agar }\end{array}$} & \multirow[t]{2}{*}{ Sex } & \multirow[t]{2}{*}{$\begin{array}{c}\text { Age (years } \\
\text { unless specified) }\end{array}$} & \multirow[t]{2}{*}{$\begin{array}{l}\text { Antimicrobial } \\
\text { agent }(s) \\
\text { administered }\end{array}$} & \multirow{2}{*}{$\begin{array}{l}\text { Length of } \\
\text { vancomycin } \\
\text { treatment } \\
\text { (days) }\end{array}$} & \multirow[t]{2}{*}{$\begin{array}{l}\text { Infection } \\
\text { source }\end{array}$} \\
\hline & & & Tei & Van & $4 \mu \mathrm{g} \mathrm{ml}^{-1}$ & $6 \mu \mathrm{g} \mathrm{ml}^{-1}$ & Tei & Van & & & & & \\
\hline CoNS13 & 2008 & S. epidermidis & 64 & 2 & + & - & 64 & 2 & M & 67 & Van, Fus & 13 & $\mathrm{CVC}$ \\
\hline CoNS25 & 2008 & S. haemolyticus & 64 & 1 & + & + & 128 & 4 & $\mathrm{~F}$ & 70 & Ceftri, Van, Gen & 5 & SSI \\
\hline CoNS46 & 2008 & S. hominis & 64 & 2 & + & - & 64 & 2 & M & 25 & Pipe, Clox, Van & 17 & Blood \\
\hline CoNS88 & 2008 & S. epidermidis & 16 & 0.5 & + & - & 16 & 1 & M & 51 & Van & 21 & $\mathrm{CVC}$ \\
\hline CoNS94 & 2008 & S. epidermidis & 16 & 0.5 & + & - & 16 & 2 & $\mathrm{~F}$ & 78 & None & 0 & CVC \\
\hline CoNS121 & 2008 & S. haemolyticus & 16 & 1 & + & - & 32 & 2 & $\mathrm{M}$ & 62 & Rif, Gen, Czid & 0 & Blood \\
\hline CoNS06 & 2009 & S. epidermidis & 128 & 2 & + & + & 128 & 4 & M & 32 & Van, Rif & 16 & Blood \\
\hline CoNS29 & 2009 & S. epidermidis & 128 & 2 & + & + & 128 & 4 & M & 3.5 & Ceftri, Van & 24 & Blood \\
\hline CoNS30 & 2009 & S. caprae & 128 & 1 & + & - & 64 & 1 & $\mathrm{~F}$ & 57 & None & 0 & $\begin{array}{l}\text { Pharynx } \\
\text { colonization }\end{array}$ \\
\hline CoNS74 & 2009 & S. haemolyticus & 128 & 2 & + & + & 128 & 2 & M & 66 & Imi, Van & 9 & CVC \\
\hline CoNS86 & 2009 & S. haemolyticus & 128 & 2 & + & - & 128 & 2 & $\mathrm{~F}$ & 82 & Imi-Cilas, Amik & 0 & Urine \\
\hline CoNS102 & 2009 & S. warneri & 128 & 2 & + & + & 128 & 6 & M & 42 & Pip/Taz, Cefti, Van & 13 & Wound \\
\hline CoNS104 & 2009 & S. hominis & 128 & 2 & + & + & 64 & 8 & $\mathrm{~F}$ & 10 & Ceftri, Van & 6 & CVC \\
\hline CoNS135 & 2009 & S. epidermidis & 64 & 0.5 & + & - & 64 & 1 & M & 64 & Van & 19 & Blood \\
\hline CoNS144 & 2009 & S. hominis & 64 & 1 & + & + & 128 & 4 & $\mathrm{~F}$ & 53 & Ceftri, Amik, Van & 12 & SSI \\
\hline CoNS147 & 2009 & S. capitis & 64 & 1 & + & - & 64 & 2 & $\mathrm{M}$ & 12 days & Mero, Amik, Van & 15 & Blood \\
\hline CoNS159 & 2009 & S. haemolyticus & 32 & 2 & + & - & 16 & 2 & $\mathrm{~F}$ & 46 & Imi, Amik, Mtz & 0 & Wound \\
\hline
\end{tabular}




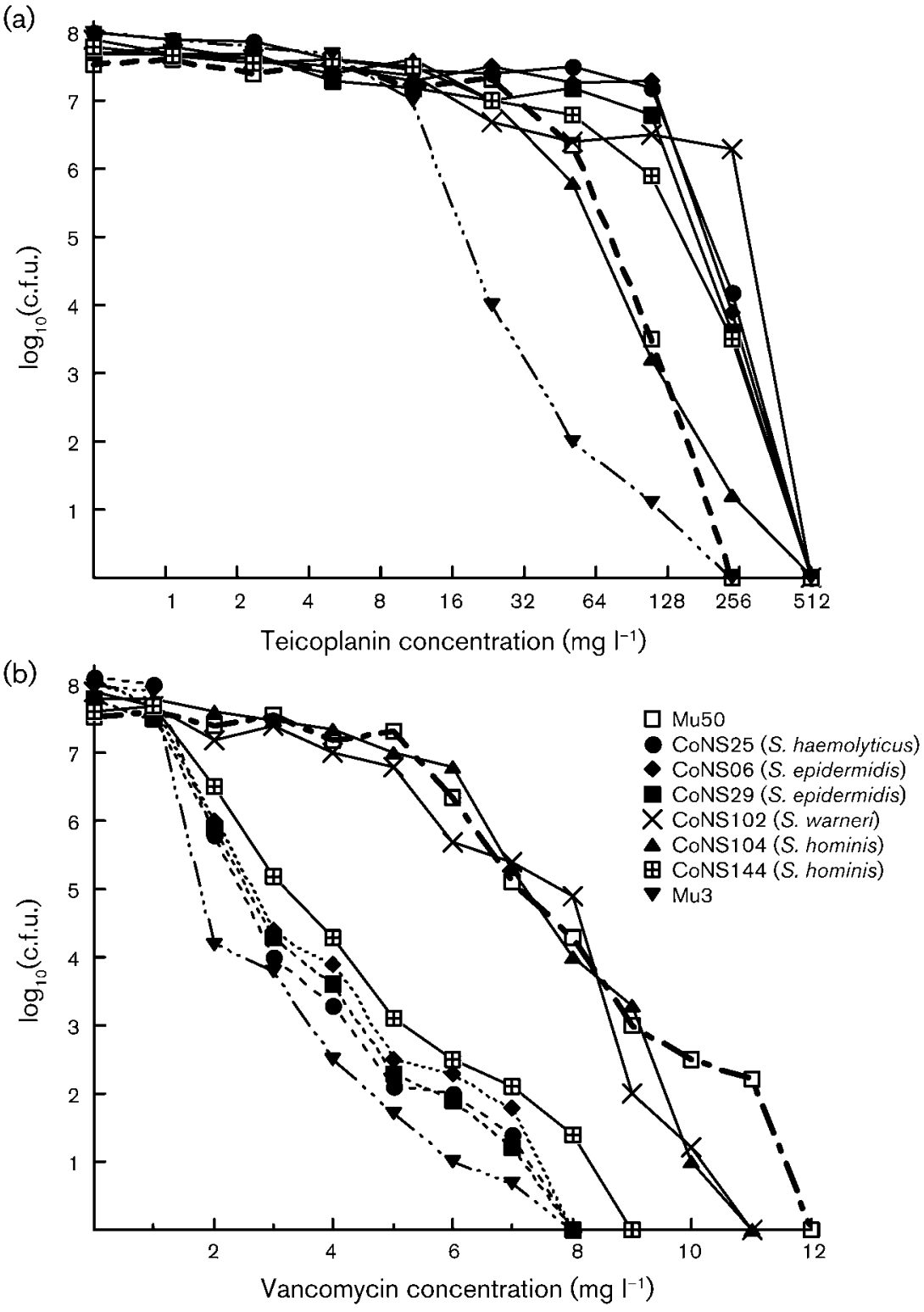

Fig. 2. Six CoNS strains that could grow on agar containing $6 \mu \mathrm{g}$ vancomycin $\mathrm{ml}^{-1}$ were selected as representative strains. Population analysis of these six representative strains in the presence of teicoplanin (a) or vancomycin (b), in comparison with Mu50 and Mu3, was performed. CoNS25, CoNS06, CoNS29 and CoNS144 showed a similar type of heteroresistance to vancomycin as Mu3. CoNS102, with a vancomycin MIC of $6 \mu \mathrm{g} \mathrm{m}^{-1}$, and CoNS104, with a vancomycin MIC of $8 \mu \mathrm{g} \mathrm{ml}^{-1}$, harboured a subpopulation of cells that could grow on agar plates containing $8 \mu \mathrm{g}$ vancomycin $\mathrm{ml}^{-1}$ at a frequency of $>0.1$ and $0.01 \%$, respectively.

empirical $\beta$-lactam therapy for sick febrile patients (Sieradzki et al., 1998). A lack of response to $\beta$-lactam therapy is often followed by addition of a glycopeptide because of the high incidence of superinfection with meticillin-resistant staphylococci.

In conclusion, the annual antimicrobial-resistance rates in CoNS strains are increasing. This poses a serious problem for medical treatment. Particularly, CoNS strains with diminished teicoplanin resistance have appeared in recent years. Antibiotic-susceptibility studies are very important from an epidemiological point of view because they allow policies to be established for adequate and rational use of antibiotics, thus making treatment more efficient and more effective. Our study summarizes the incidence and evolution of teicoplanin resistance among CoNS over a period of 6 years. Both the teicoplanin MIC values and the number of non-susceptible strains increased from 2008 to 2009 (six CoNS strains with MIC values of 16 and $64 \mu \mathrm{g} \mathrm{ml}^{-1}$ in 2008; seven CoNS strains with a MIC value of $128 \mu \mathrm{g} \mathrm{ml}^{-1}$ in 2009). More CoNS species were also observed in the teicoplanin-non-susceptible CoNS strains in 2009 than in 2008. A one-step vancomycin agar selection experiment and subsequent population analysis revealed potentially vancomycin-resistant subpopulations that have been selected from the teicoplanin-non-susceptible strains. Cell culture of CoNS102 and CoNS104, which are capable of growing at $6 \mu \mathrm{g}$ vancomycin $\mathrm{ml}^{-1}$, showed stable vancomycin MICs of 6 and $8 \mu \mathrm{g} \mathrm{ml}^{-1}$, respectively. $\operatorname{van} A, \operatorname{van} B$ and $\operatorname{van} C$ genes were not detected in these strains by PCR amplification, suggesting that glycopeptide resistance in these strains occurred endogenously, rather than via 
acquisition of transferable plasmids, transposons or closely related elements. Further studies on the ultrastructural morphology, glycopeptide-binding capacity, membrane proteins, cell-wall composition and susceptibility to cellactive antibiotics and enzymes are needed to elucidate the mechanism of glycopeptide resistance in these strains.

As the use of teicoplanin has been limited in the Second Affiliated Hospital of China Medical University, the emergence of teicoplanin resistance among CoNS might be the result of selective pressure from the frequent use of vancomycin. Our results show that 12 patients received vancomycin as an antimicrobial-chemotherapy drug, and eight of the 12 patients received $\beta$-lactam antibiotics before or concomitantly with vancomycin, verifying the possibility that prior use of $\beta$-lactams might serve to induce the expression of vancomycin resistance. The sequential use of vancomycin could then exert its effects through selection of vancomycin-resistant subclones in vivo. This is a cause for clinical concern, as vancomycin is still the reasonable choice for the treatment of severe infections due to multiple-resistant CoNS. There is a need for surveillance of nosocomial isolates of CoNS for resistance to glycopeptides.

\section{ACKNOWLEDGEMENTS}

The authors thank Professors Keiiti Hiramatsu and Teruyo Ito (Juntendo University) for providing VRSA strain Mu50 and heteroVRSA strain Mu3. We also thank Professors Xiao Song Qin and Ji Mei Sun (Department of Clinical Microbiology, Second Affiliated Hospital of China Medical University) for providing CoNS strains isolated from clinical specimens. This work was supported by the National Natural Science Foundation (no. 30972520) and the Ministry of Education in China [no. (2009) 1001].

\section{REFERENCES}

Bourgeois, I., Pestel-Caron, M., Lemeland, J. F., Pons, J. L. \& Caron, F. (2007). Tolerance to the glycopeptides vancomycin and teicoplanin in coagulase-negative staphylococci. Antimicrob Agents Chemother 51, 740-743.

Boyce, J. M. (2004). Coagulase-negative staphylococci. In Hospital Epidemiology and Infection Control, 3rd edn, pp. 495-516. Edited by C. G. Mayhall. Philadelphia, PA: Lippincott Williams \& Wilkins.

Casey, A. L., Lambert, P. A. \& Elliott, T. S. J. (2007). Staphylococci. Int J Antimicrob Agents 29 (Suppl. 3), S23-S32.

Cervera, C., Almela, M., Martínez-Martínez, J. A., Moreno, A. \& Miró, J. M. (2009). Risk factors and management of Gram-positive bacteraemia. Int J Antimicrob Agents 34 (Suppl. 4), S26-S30.

CLSI (2010). Performance Standards for Antimicrobial Susceptibility Testing, 19th Informational Supplement, M100-S19. Wayne, PA: Clinical and Laboratory Standards Institute.

Cremniter, J., Slassi, A., Quincampoix, J. C., Sivadon-Tardy, V., Bauer, T., Porcher, R., Lortat-Jacob, A., Piriou, P., Judet, T. \& other authors (2010). Decreased susceptibility to teicoplanin and vancomycin in coagulase-negative staphylococci isolated from orthopedicdevice-associated infections. J Clin Microbiol 48, 1428-1431.

Dubois, D., Leyssene, D., Chacornac, J. P., Kostrzewa, M., Schmit, P. O., Talon, R., Bonnet, R. \& Delmas, J. (2010). Identification of a variety of
Staphylococcus species by matrix-assisted laser desorption ionizationtime of flight mass spectrometry. J Clin Microbiol 48, 941-945.

Ferreira, R. B., Iorio, N. L., Malvar, K. L., Nunes, A. P., Fonseca, L. S., Bastos, C. C. \& Santos, K. R. (2003). Coagulase-negative staphylococci: comparison of phenotypic and genotypic oxacillin susceptibility tests and evaluation of the agar screening test by using different concentrations of oxacillin. J Clin Microbiol 41, 3609-3614.

Gazzola, S. \& Cocconcelli, P. S. (2008). Vancomycin heteroresistance and biofilm formation in Staphylococcus epidermidis from food. Microbiology 154, 3224-3231.

Huebner, J. \& Goldmann, D. A. (1999). Coagulase-negative staphylococci: role as pathogens. Annu Rev Med 50, 223-236.

Hung, K. H., Yan, J. J., Lu, Y. C., Chen, H. M. \& Wu, J. J. (2011). Evaluation of discrepancies between oxacillin and cefoxitin susceptibility in coagulase-negative staphylococci. Eur J Clin Microbiol Infect Dis 30, 785-788.

Kristóf, K., Kocsis, E., Szabó, D., Kardos, S., Cser, V., Nagy, K., Hermann, P. \& Rozgonyi, F. (2011). Significance of methicillinteicoplanin resistant Staphylococcus haemolyticus in bloodstream infections in patients of the Semmelweis University hospitals in Hungary. Eur J Clin Microbiol Infect Dis 30, 691-699.

Lorenzo-Díaz, F., Delgado, T., Reyes-Darias, J. A., Flores, C., Méndez-Alvarez, S., Villar, J., Sierra, A. \& Claverie-Martín, F. (2004). Characterization of the first VanB vancomycin-resistant Enterococcus faecium isolated in a Spanish hospital. Curr Microbiol 48, 199-203.

Ma, X. X., Sun, D. D., Hu, J., Wang, E. H. \& Luo, E. J. (2011). Epidemiological and molecular characterization of Staphylococcus haemolyticus strains, from a hematology ward, with decreased susceptibility to glycopeptides. Can J Microbiol 57, 476-484.

Maniati, M., Petinaki, E., Spiliopoulou, I., Kontos, PetropoulouMylona, D., Spaliara, L., Malamou-Lada, H., Koutsia-Carouzou, C. \& Maniatis, A. N. (2005). Rapid increase in numbers of Staphylococcus epidermidis strains with reduced susceptibility to teicoplanin in Greece. Int J Antimicrob Agents 25, 346-348.

Matsuhashi, M., Song, M. D., Ishino, F., Wachi, M., Doi, M., Inoue, M., Ubukata, K., Yamashita, N. \& Konno, M. (1986). Molecular cloning of the gene of a penicillin-binding protein supposed to cause high resistance to $\beta$-lactam antibiotics in Staphylococcus aureus. J Bacteriol 167, 975-980.

Natoli, S., Fontana, C., Favaro, M., Bergamini, A., Testore, G. P., Minelli, S., Bossa, M. C., Casapulla, M., Broglio, G. \& other authors (2009). Characterization of coagulase-negative staphylococcal isolates from blood with reduced susceptibility to glycopeptides and therapeutic options. BMC Infect Dis 9, 83.

Otto, M. (2009). Staphylococcus epidermidis - the 'accidental' pathogen. Nat Rev Microbiol 7, 555-567.

Palazzo, I. C., Araujo, M. L. \& Darini, A. L. (2005). First report of vancomycin-resistant staphylococci isolated from healthy carriers in Brazil. J Clin Microbiol 43, 179-185.

Périchon, B. \& Courvalin, P. (2009). VanA-type vancomycin-resistant Staphylococcus aureus. Antimicrob Agents Chemother 53, 4580-4587.

Sieradzki, K., Villari, P. \& Tomasz, A. (1998). Decreased susceptibilities to teicoplanin and vancomycin among coagulase-negative methicillin-resistant clinical isolates of staphylococci. Antimicrob Agents Chemother 42, 100-107.

Srinivasan, A., Dick, J. D. \& Perl, T. M. (2002). Vancomycin resistance in staphylococci. Clin Microbiol Rev 15, 430-438.

Stefani, S. \& Varaldo, P. E. (2003). Epidemiology of methicillinresistant staphylococci in Europe. Clin Microbiol Infect 9, 1179-1186.

Tacconelli, E., Tumbarello, M., Donati, K. G., Bettio, M., Spanu, T., Leone, F., Sechi, L. A., Zanetti, S., Fadda, G. \& Cauda, R. (2001). 
Glycopeptide resistance among coagulase-negative staphylococci that cause bacteremia: epidemiological and clinical findings from a casecontrol study. Clin Infect Dis 33, 1628-1635.

Trueba, F., Garrabe, E., Hadef, R., Fabre, R., Cavallo, J. D., Tsvetkova, K. $\&$ Chesneau, O. (2006). High prevalence of teicoplanin resistance among Staphylococcus epidermidis strains in a 5-year retrospective study. J Clin Microbiol 44, 1922-1923.
Tschudin Sutter, S., Frei, R., Dangel, M., Gratwohl, A., Bonten, M. \& Widmer, A. F. (2010). Not all patients with vancomycinresistant enterococci need to be isolated. Clin Infect Dis 51, 678683.

Zong, Z. Y., Peng, C. H. \& Lü, X. J. (2011). Diversity of SCCmec elements in methicillin-resistant coagulase-negative staphylococci clinical isolates. PLoS One 6, e20191. 